\title{
NOVEL RHOGAP INDEPENDENT PATHWAY OF TUMOR SUPPRESSOR DLC1 REGULATES CANCER INVASION AND METASTASIS
}

Frankie Chi Fat Ko, Yin-Shan Yeung, Xiaowen Mao, and Judy Wai Ping Yam

\author{
University of Hong Kong, Pokfulam, Hong Kong
}

Hepatocellular carcinoma (HCC) is the fifth most common cancer worldwide. Deleted in Liver Cancer 1 (DLC1) is a tumor suppressor gene critically involved in hepatocarcinogenesis. DLC1 is a Rho GTPase activating protein (RhoGAP), which serves as the negative regulator of Rho proteins. Rho proteins are important in remodeling of actin cytoskeleton, transcription regulation, cell proliferation, tumorigenesis and metastasis. Apart from RhoGAP activity, the inhibitory activity of DLC1 is also dependent on its proper focal adhesion localization. It has been found that tensin proteins are responsible for directing DLC1 to the focal adhesions via their interaction which is important to the tumor suppression activity of DLC1. Recently, we identified that cAMP/Protein Kinase A (PKA) could induce DLC1 dimerization by phosphorylation for activation. PKC and PKD had also been demonstrated to phosphorylate DLC1 and modulate the nuclear shuttling and RhoGAP activity of DLC1. Our previous study also revealed that PKB/Akt phosphorylates DLC1 and subsequently attenuates the tumor suppressive activity of DLC1 through a RhoGAP independent pathway.

Our data revealed that DLC1 Ser-567 phosphorylation affects its binding with Akt, prompting us to further search for other DLC1 interacting partners which are specifically regulated by Ser-567 phosphorylation. We performed differential co-immunoprecipitation assay to compare protein expressions of phospho-defective mutant (S567A) and phosphomimetic mutant (S567D) of DLC1. Co-immunoprecipitated proteins were then subjected to mass spectrometry for protein identity. Among all interacting candidates, we are interested to characterize the midline family genes: midline-1 (MID-1) and midline-2 (MID-2). MID-1 mutation is associated with embryonic developmental defect, X-linked Opitz G/BBB syndrome (XLOS). It is important for epithelialmesenchymal differentiation, cell migration and adhesion, and apoptosis during embryogenesis. MID1 protein is a microtubule-associated ubiquitin E3 ligase. MID1 targets catalytic subunit of protein phosphatase 2A (PP2Ac) for ubiquitination by binding with $\alpha 4$. In HCC, $\alpha 4$ is highly expressed. From our analysis, MID-1/2 proteins interact with S567A but not with S567D, suggesting that Akt phosphorylation abolishes the interaction between DLC1 and MID-1/2. Thus, it would be tempting to investigate how DLC1 affects MID/ $\alpha 4 / \mathrm{PP} 2 \mathrm{~A}$ complex in controlling epithelial-mesenchymal transition, cell-cycle checkpoint and inducing apoptosis. Our initial investigation has provided evidence that MID-1/2 are novel interacting proteins of DLC1 and their interaction is mediated by phosphorylation of DLC1 at Ser-567. In this study, we aim to investigate the functional significance of DLC1 and MID-1/2 and how DLC1 modulates the functions of MID/ $44 / \mathrm{PP} 2 \mathrm{~A}$ complex in liver cancer. The functional roles of DLC1 and MID-1/2 interaction in HCC metastasis will also be discussed. 